\title{
Evaluación de Diferentes Concentraciones de Tricaína (MS-222) en el Transporte de Chitas (Anisotremus scapularis) Juveniles
}

\author{
Evaluation of Different Tricaine (MS-222) Concentrations on the Transport \\ of Juvenile Peruvian Grunt (Anisotremus scapularis)
}

\author{
Maryandrea Rosado $^{1,2,3}$, Jhon Dionicio ${ }^{1}$, Arturo Aguirre-Velarde ${ }^{1}$
}

\section{Resumen}

Se evaluó el uso de la tricaína (MS-222) como agente sedante para uso en el transporte de Anisotremus scapularis juveniles . Para ello, se realizó un transporte simulado de 24 horas a dos temperaturas $\left(15\right.$ y $\left.19^{\circ} \mathrm{C}\right)$ usando concentraciones del anestésico de 0 , 15,20 y $25 \mathrm{mg} . \mathrm{L}^{-1}$ en bolsas de polietileno ( $50 \%$ agua de mar - $50 \%$ oxígeno puro). Se monitoreó, tanto al inicio como al final de la simulación, la temperatura, saturación de oxígeno disuelto, $\mathrm{pH}$ y tasa de excreción individual de amonio. En los peces se evaluó la pérdida de equilibrio (\%) y sobrevivencia (\%) al término de la simulación y luego de un tiempo de recuperación de 24 horas adicionales. Los resultados muestran que el uso de tricaína favorece la disminución de la excreción de amonio; sin embargo, dosis superiores a $20 \mathrm{mg} . \mathrm{L}^{-1}$ de tricaína incrementan considerablemente la pérdida de equilibrio. Además, el efecto de sedación es más intenso a temperaturas más bajas $\left(15^{\circ} \mathrm{C}\right)$. Por otro lado, el $\mathrm{pH}$ del agua se ve afectado mostrando una disminución promedio de 1.44 durante la simulación del transporte en todos los tratamientos. Con base a los resultados se recomienda el transporte de juveniles de chita a $19{ }^{\circ} \mathrm{C}$ con uso de tricaína a $15 \mathrm{mg} . \mathrm{L}^{-1}$.

Palabras clave: fisiología, tricaína, sedación, transporte de peces, maricultura, chita

\section{AbSTRACT}

The use of tricaine (MS-222) as sedative agent for transporting of Anisotremus scapularis juveniles was evaluated. A simulated transport of 24 hours was performed at two temperatures $\left(15\right.$ and $\left.19^{\circ} \mathrm{C}\right)$ using the anaesthetic concentrations of $0,15,20$ and 25 mg. $\mathrm{L}^{-1}$ in polyethylene bags ( $50 \%$ seawater $-50 \%$ pure oxygen). At the beginning and the

\footnotetext{
${ }^{1}$ Laboratorio de Ecofisiología Acuática, Instituto del Mar del Perú (IMARPE), Callao, Perú

${ }^{2}$ E-mail: mrosado@imarpe.gob.pe

${ }^{3}$ Fuente de financiamiento: PpR Ordenamiento y Desarrollo de la Acuicultura (DGIA-IMARPE)
}

Recibido: 28 de abril de 2016

Aceptado para publicación: 15 de agosto de 2016 
end of the simulation temperature, dissolved oxygen saturation, $\mathrm{pH}$ and ammonia excretion rate were monitored. On fishes, the loss of balance (\%) and survival (\%) were monitored at the end of the simulation and after an additional recovery time of 24 hours. The results showed that use of tricaine favours the reduction of ammonia excretion, however, tricaine doses over $20 \mathrm{mg} . \mathrm{L}^{-1}$ significantly increase the loss of balance in fish. Furthermore, the sedating effect is magnified at lower temperature $\left(15^{\circ} \mathrm{C}\right)$. On the other hand, the water $\mathrm{pH}$ is affected showing an average decrease of 1.44 during the transport simulation in all treatments. Based on the results, transport of Peruvian grunt juveniles using tricaine in a dose of at $15 \mathrm{mg} \cdot \mathrm{L}^{-1}$ at $19{ }^{\circ} \mathrm{C}$ is recommended.

Key words: physiology, tricaine, sedation, live fish transport, mariculture, Peruvian grunt

\section{INTRODUCCCIÓN}

La chita (Anisotremus scapularis Tschudi, 1846) es una especie de importancia comercial en las costas del Perú, alcanzando desembarques anuales de hasta $274 \mathrm{t}$ (Produce, 2015). Este pez marino, también conocido como Sargo (Peruvian grunt, en inglés) pertenece a la familia Haemulidae, distribuyéndose desde Manta (Ecuador) hasta Antofagasta (Chile), incluidas las Islas de Cocos y Galápagos (Chirichigno y Vélez, 1998). Presentan sexos separados, llegando a medir hasta $40 \mathrm{~cm}$ (Allen y Robertson, 1994) y $896 \mathrm{~g}$ de peso (Medina et al., 2004). Tienen el cuerpo lateralmente comprimido, labios gruesos, hocico romo, aleta dorsal con 13 espinas y de 16 a 17 radios, aleta anal con 3 espinas y de 12 a 13 radios. A. scapularis es de color gris plateado con una mancha negra, tanto en la axila de la aleta pectoral como en la base del último radio dorsal y anal (Chirichigno y Vélez, 1998).

Actualmente, la acuicultura peruana está orientada al cultivo de unas pocas especies, siendo las más desarrolladas la concha de abanico, langostino, trucha y tilapia (FAO, 2010). El Gobierno Peruano viene promoviendo la investigación e innovación de técnicas para el cultivo de chita, con el fin de brindar nuevas alternativas productivas y diversificar la maricultura. Sin embargo, los estudios que se han realizado sobre chita son aún escasos, conociéndose su distribución, taxono- mía y características externas (ver Chirichigno y Vélez, 1998), la descripción de algunas especies parasitarias que presenta la chita en la zona central y sur del litoral (ver Ruelas y Cordova, 1995; Iannacone y Alvariño, 2009, 2012; Chero et al., 2014), e información de desembarques pesqueros (ver Produce, 2015).

Un punto crítico necesario para la producción acuícola es el transporte de larvas, alevines o juveniles de la eclosería a los centros de engorde. Durante el transporte se producen cambios en la calidad de agua; entre ellos, aumento en la concentración de gas carbónico, disminución del $\mathrm{pH}$ y aumento de la concentración de amoniaco y de sólidos en suspensión (Kubitza, 2009). Esta degradación de la calidad de agua de transporte afecta la sobrevivencia de los peces (Hettiarachchi y Senadheera, 1999), reduciendo la productividad. Con el fin de evitar estos efectos adversos, los acuicultores utilizan una variedad de métodos, tales como la suspensión del alimento antes del transporte, disminución de la temperatura y uso de anestésicos (Hettiarachchi y Senadheera, 1999).

El uso de anestésicos con el fin de reducir la actividad metabólica y la respuesta de estrés en peces durante el transporte se ha incrementado en los últimos años (Summerfelt y Smith, 1990; Carter et al., 2011). Dentro de los anestésicos más usados se encuentran 2-fenoxietanol, aceite de clavo, sulfato de quinaldina, benzocaína y 
metasulfonato de tricaína (MS-222). Sin embargo, algunos de estos han sido clasificados como tóxicos para varias especies acuáticas, lo cual implica una rigurosa evaluación de sus efectos antes de recomendar su uso como alternativa para reducir el estrés durante la manipulación y transporte de peces (Velasco et al., 2008).

La tricaína (MS-222) se clasifica como un anestésico local sintético tipo éster y se utiliza comúnmente en la industria pesquera y acuícola (Sato et al., 2000; Carter et al., 2011). Este anestésico es usado con éxito en las especies de las familias Ictaluridae (peces gato), Salmonidae (salmón, trucha, trucha ártica, pez blanco y tímalo), Esocidae y Percidae (Carter et al., 2011). En este contexto, el objetivo del presente trabajo fue evaluar el uso de diferentes dosis de tricaína (MS222) en el transporte de juveniles de chita, con el fin de aportar al conocimiento técnico que permita un transporte eficiente, con alto porcentaje de sobrevivencia y, de esta manera, impulsar el desarrollo del cultivo de peces marinos en el Perú.

\section{Materiales y Métodos}

\section{Concentraciones de Tricaína (MS -222)}

Se realizó una prueba preliminar para determinar las concentraciones de tricaína que podrían ser utilizadas durante la prueba de transporte simulado. Se emplearon juveniles de $A$. scapularis de $6-7 \mathrm{~cm}$, previamente aclimatados en tanques de $300 \mathrm{~L}$ de capacidad, con aireación constante y temperatura a $19{ }^{\circ} \mathrm{C}$. En esta prueba se utilizaron cinco acuarios de vidrio de $5 \mathrm{~L}$ de capacidad (cuatro tratamientos y un control sin anestésico) con aireación constante y temperatura a $19^{\circ} \mathrm{C}$. En los acuarios con tratamiento de anestésico se disolvió tricaína suficiente para lograr concentraciones de 20,23, 26, 29 $\mathrm{mg} . \mathrm{L}^{-1}$. Luego, los peces fueron colocados en los acuarios y expuestos a las condiciones experimentales durante 24 horas.
En este periodo se evaluó en cada acuario, tanto al inicio como al final de exposición al anestésico, la Frecuencia Opercular (FO/min) de tres individuos con un contómetro manual en lapsos de 30 segundos, el porcentaje de individuos que presentaban Pérdida de Equilibrio (PE, \%), Respuesta al Estimulo Mecánico (REM) y Sobrevivencia (\%).

Como resultado de la prueba preliminar (Cuadro 1), se observó que $29 \mathrm{mg} . \mathrm{L}^{-1} \mathrm{de}$ tricaína sobrepasó los límites de sedación para juveniles de chita; presentando el mayor porcentaje de PE (>50\%), donde incluso luego de 24 horas se podía observar individuos con nado irregular. La FO se mantuvo elevada en comparación con tratamientos de concentraciones más bajas de anestésico, además de presentar la menor sobrevivencia (40\%) tras la exposición. Asimismo, a $26 \mathrm{mg} . \mathrm{L}^{-1} \mathrm{de}$ tricaína se observó individuos que presentaron pérdida del equilibrio (20\%), sobrepasando el límite deseado de sedación de juveniles de chita para el transporte; sin embargo, la sobrevivencia fue de $100 \%$ al término de la exposición.

\section{Material Biológico}

Se utilizaron juveniles de $A$. scapularis con 6-9 cm de rango de longitud total, obtenidos de desoves en cautiverio en el Instituto del Mar del Perú (IMARPE). Previo al experimento, los peces fueron aclimatados en dos tanques de $2 \mathrm{~m}^{3}$, equipados con sistema de recirculación, aireación constante y fotoperiodo natural. En cada tanque fueron colocados 85 peces. La temperatura inicial del agua de los tanques fue de $19{ }^{\circ} \mathrm{C}$. En uno de los tanques la temperatura fue disminuida a $15^{\circ} \mathrm{C}$ en un lapso de 5 días. Las temperaturas de 15 y $19^{\circ} \mathrm{C}$ en los tanques se mantuvieron constantes por 7 días antes del inicio de la exposición al anestésico. Durante la aclimatación, los ejemplares fueron alimentados una vez al día con dieta comercial para peces marinos OTOHIME EP2 de $2 \mathrm{~mm}$ de diámetro. La alimentación fue suspendida 24 horas antes de la simulación de transporte. 
Cuadro 1. Resultados de frecuencia opercular (FO), sobrevivencia, pérdida de equilibrio (PE) y respuesta al estímulo mecánico (REM) en juveniles de A. scapularis en la prueba preliminar de determinación de concentraciones de tricaína (MS-222) al inicio y a $24 \mathrm{~h}$ de exposición

\begin{tabular}{cccccc}
\hline Tiempo (h) & $\begin{array}{c}\text { Tricaína } \\
\left(\mathrm{mg} . \mathrm{L}^{-1}\right)\end{array}$ & $\begin{array}{c}\text { FO } \\
(/ \mathrm{min})\end{array}$ & $\begin{array}{c}\text { Sobrevivencia } \\
(\%)\end{array}$ & $\begin{array}{c}\text { PE } \\
(\%)\end{array}$ & REM \\
\hline \multirow{3}{*}{0} & Control & 32.6 & 100 & 0 & Presente \\
& 20 & 33.2 & 100 & 0 & Presente \\
& 23 & 30.6 & 100 & 0 & Presente \\
& 26 & 32.0 & 100 & 20 & Presente \\
& 29 & 32.6 & 100 & 60 & Ausente \\
& & & & & \\
& Control & 24.0 & 100 & 0 & Presente \\
& 20 & 28.0 & 100 & 0 & Presente \\
& 23 & 25.2 & 100 & 0 & Presente \\
& 26 & 26.6 & 100 & 0 & Presente \\
& 29 & 31.0 & 40 & 20 & Presente \\
\hline
\end{tabular}

\section{Prueba de Simulación de Transporte}

Se utilizó un diseño factorial cruzado 2 $\mathrm{x} 4$, teniendo dos temperaturas $\left(15\right.$ y $\left.19^{\circ} \mathrm{C}\right)$ y cuatro concentraciones de tricaína (control, 15, 20, 25 mg. $\left.\mathrm{L}^{-1}\right)$, sumando un total de ocho tratamientos, cada uno con tres repeticiones (Cuadro 2). Las concentraciones de tricaína utilizadas son el resultado de la prueba preliminar descrita anteriormente. Para la simulación de transporte, se utilizaron bolsas de polietileno transparente con capacidad de $10 \mathrm{~L}$. Las bolsas fueron llenadas con $5 \mathrm{~L}$ de agua de mar a 15 y $19^{\circ} \mathrm{C}$ según el caso. En las bolsas con tratamiento de anestésico, se disolvió tricaína suficiente para lograr concentraciones de 15, 20 y $25 \mathrm{mg} . \mathrm{L}^{-1}$. Se colocaron siete peces en cada bolsa, teniendo en cuenta la temperatura de transporte igual a la temperatura de aclimatación. Se inyectó $5 \mathrm{~L}$ aproximadamente de oxígeno puro industrial en las bolsas (Figura 1a) y fueron cerradas en forma hermética utilizando bandas elásticas (ver Woynarovich y Horváth [1981] para descripción detallada de la técnica) (Figura 1b).
Para mantener constante la temperatura del agua, las bolsas fueron colocadas en los tanques con agua termorregulados a $15 \mathrm{y}$ $19^{\circ} \mathrm{C}$ de temperatura, respectivamente. Las condiciones de transporte simulado fueron mantenidas por un periodo de 24 horas.

Cuadro 2. Nomenclatura de los tratamientos utilizados en la simulación de transporte de juveniles de chita

\begin{tabular}{ccc}
\hline $\begin{array}{c}\text { Temperatura } \\
\left({ }^{\circ} \mathrm{C}\right)\end{array}$ & $\begin{array}{c}\text { Tricaína } \\
\left(\mathrm{mg}^{-1} \mathrm{~L}^{-1}\right)\end{array}$ & $\begin{array}{c}\text { Nomenclatura } \\
\text { de } \\
\text { tratamientos }\end{array}$ \\
\hline 15 & $\begin{array}{c}\text { Control } \\
15 \mathrm{C}-0\end{array}$ & $15 \mathrm{C}-15$ \\
& 15 & $15 \mathrm{C}-20$ \\
& 20 & $15 \mathrm{C}-25$ \\
19 & 25 & $19 \mathrm{C}-0$ \\
& Control & $19 \mathrm{C}-15$ \\
& 15 & $19 \mathrm{C}-20$ \\
& 20 & $19 \mathrm{C}-25$ \\
\hline
\end{tabular}



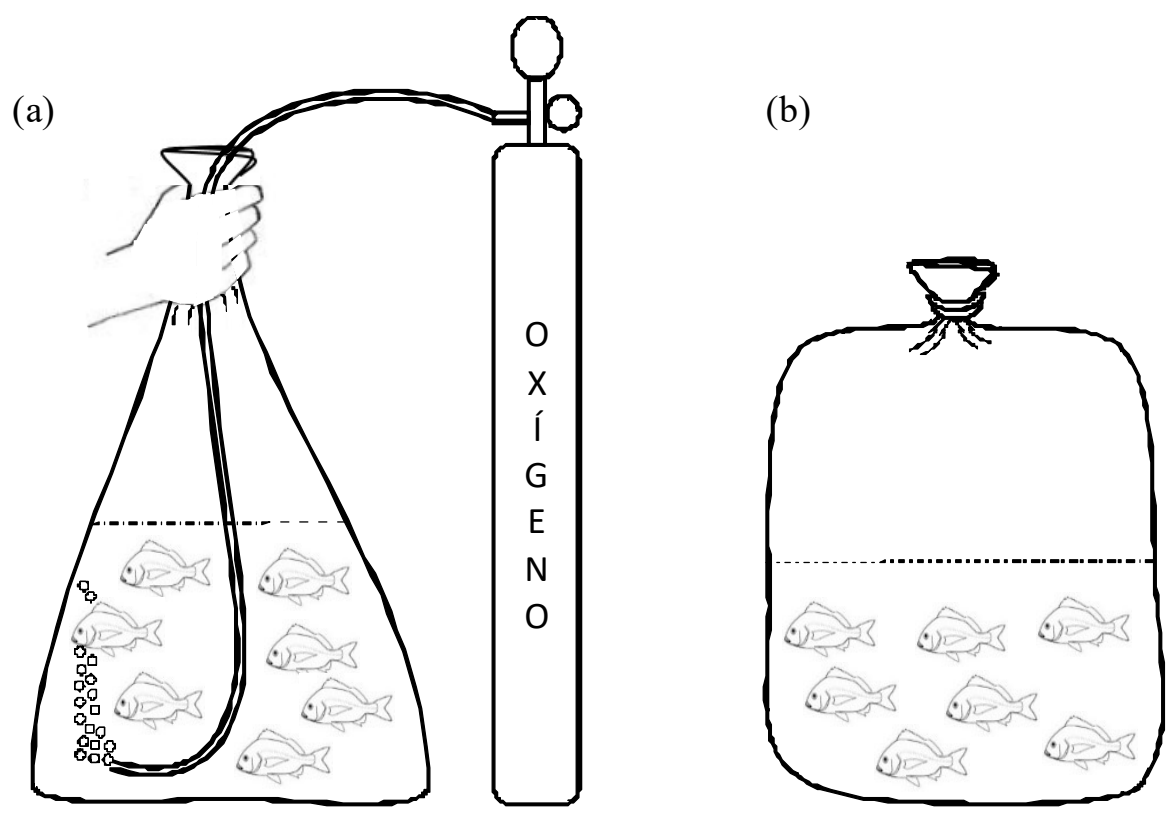

Figura 1. Descripción de llenado de bolsas utilizadas en la simulación de transporte de chita. Se muestra detalle de la inyección de $\mathrm{O}_{2}$ (a) y bolsa sellada herméticamente conteniendo $5 \mathrm{~L}$ agua y 7 peces (b)

Luego del transporte simulado, los peces fueron transferidos a jaulas flotantes dentro de los tanques termorregulados a las temperaturas de 15 y $19{ }^{\circ} \mathrm{C}$. Las tres réplicas de cada tratamiento se colocaron en una sola jaula a la temperatura correspondiente. Los peces fueron observados por 24 horas adicionales para evaluar su recuperación y sobrevivencia (evaluaciones luego de 48 horas de iniciada la simulación de transporte).

\section{Monitoreo de Condiciones Experimen- tales}

Se evaluaron las condiciones de transporte al inicio y final de la simulación en cada una de las bolsas. La temperatura $\left({ }^{\circ} \mathrm{C}\right)$, la saturación de oxígeno disuelto (\%) y el pH fueron evaluados con un multiparámetro WTW Multi 3430.

La concentración de amonio $(\mu \mathrm{M})$ fue determinada por fluorometría con el equipo 10-AU Tuner Designs. Para este fin, se colectaron $40 \mathrm{ml}$ de agua en frascos de vidrio con tapa hermética. La tasa de excreción de amonio individual $\left(\mu \mathrm{M}\right.$. ind $\left.\mathrm{d}^{-1} . \mathrm{h}^{-1}\right)$ se estimó con la fórmula EAI $=\left[\left(\mathrm{CA}_{\text {final }}-\mathrm{CA}_{\text {inicial }}\right) /\right.$ $\left.\mathrm{n}_{\text {ind }}\right] / \mathrm{t}$, donde EAI: tasa de excreción de amonio individual $\left(\mu \mathrm{M}\right.$. ind $\left.{ }^{-1} \cdot \mathrm{h}^{-1}\right) ; \mathrm{CA}_{\text {final }}$ : concentración de amonio en la bolsa al finalizar la simulación de transporte $(\mu \mathrm{M}) ; \mathrm{CA}_{\text {inicial }}$ : concentración de amonio al inicio de la simulación de transporte $(\mu \mathrm{M}) ; \mathrm{n}_{\text {ind }}$ : número de peces vivos dentro de la bolsa al finalizar la simulación de transporte; $\mathrm{y}$ t: tiempo de duración de la simulación de transporte (24 horas).

Además, se registraron los porcentajes de PE (\%) y de sobrevivencia (\%) de los peces en cada réplica (bolsa de simulación de transporte). Estas observaciones se realizaron en cada una de las bolsas al finalizar la simulación de transporte y al finalizar el periodo de recuperación en las jaulas flotantes.

\section{Evaluación de Tratamientos}

Con el fin de identificar las mejores condiciones de transporte, se evaluaron los índi- 
ces de EAI, PE y sobrevivencia. Para ello se estimaron las medias de cada índice y se compararon los errores estándar de cada índice alrededor de la media entre los tratamientos. Se implementó un sistema de puntaje, en el cual se otorgó el puntaje de «1» al «mejor tratamiento», según sea el caso del índice evaluado. De igual manera se otorgó el puntaje de «1» a aquellos tratamientos cuyo índice promedio se encontrara dentro del rango del error estándar del «mejor tratamiento». Un puntaje de « $\ll »$ fue otorgado a aquellos tratamientos cuyo índice promedio se encontrara por debajo o por encima, según el caso, del rango de error estándar del «mejor tratamiento». Por último, se sumaron puntos de cada índice para cada tratamiento. El tratamiento que presentó mayor puntaje fue recomendado para el transporte de juveniles de chita con MS-222 a las temperaturas evaluadas.

\section{Resultados}

\section{Condiciones Experimentales}

La temperatura del agua se mantuvo relativamente constante $\left(14 \pm 0.7\right.$ y $\left.19 \pm 0.4^{\circ} \mathrm{C}\right)$ en los estanques durante la simulación de transporte y posterior recuperación de los peces. El oxígeno disuelto se encontró supersatu-rado, con valores mayores al $120 \%$ al interior de las bolsas, tanto al inicio como al finalizar la simulación. En cuanto a los valores de $\mathrm{pH}$ (Cuadro 3), se observó una disminución promedio para todos los tratamientos de cerca de 1.44 .

En los tratamientos $15 \mathrm{C}-20,15 \mathrm{C}-25$, 19C-15 y 19C-20, la producción de amonio se mantuvo relativamente baja con valores alrededor de los $0.008 \mu \mathrm{M}$. ind ${ }^{-1} \cdot \mathrm{h}^{-1}$. Valores de excreción ligeramente más elevados fueron observados en el tratamiento $15 \mathrm{C}-15 \mathrm{con}$ una tasa de $0.009 \mu \mathrm{M}$. ind $^{-1} \cdot h^{-1}$. Los tratamientos $15 \mathrm{C}-0$ y $19 \mathrm{C}-0$ presentaron las tasas de excreción de amonio más altas con promedios de 0.012 y $0.018 \mu \mathrm{M}$. ind ${ }^{-1} \cdot h^{-1}$, res-
Cuadro 3. Valores de $\mathrm{pH}$ en cada tratamiento (concentración de tricaína (MS-222 y temperatura del estanque) al inicio y al final de la prueba

\begin{tabular}{ccc}
\hline \multirow{2}{*}{ Tratamiento } & \multicolumn{2}{c}{$\mathrm{pH}$} \\
\cline { 2 - 3 } & Inicial & Final \\
\hline $15 \mathrm{C}-0$ & & 6.49 \\
$15 \mathrm{C}-15$ & \multirow{2}{*}{7.91} & 6.44 \\
$15 \mathrm{C}-20$ & & 6.46 \\
$15 \mathrm{C}-25$ & & 6.47 \\
$19 \mathrm{C}-0$ & & 6.37 \\
$19 \mathrm{C}-15$ & & 6.41 \\
$19 \mathrm{C}-20$ & 7.81 & 6.41 \\
$19 \mathrm{C}-25$ & & 6.37 \\
\hline
\end{tabular}

pectivamente. La excreción en el tratamiento $19 \mathrm{C}-25$ no fue calculada por presentar una alta mortalidad ( $>50 \%$ ) (Figura $2 a)$.

En cuanto a la capacidad de natación, en los tratamientos $19 \mathrm{C}-0$ y $19 \mathrm{C}-15$ se registraron bajos porcentajes de pérdida de equilibrio, con un promedio del $14 \%$ para ambos casos. El tratamiento $15 \mathrm{C}-0$ con un promedio de $29 \%$ también presentó un porcentaje relativamente bajo de individuos con pérdida de equilibrio. Los tratamientos 15C-15, 19C20 y $19 \mathrm{C}-25$ sobrepasaron el $50 \%$ de individuos con pérdida de equilibrio, mientras que los tratamientos $15 \mathrm{C}-20$ y $15 \mathrm{C}-25$ alcanzaron $100 \%$ (Figura 2b).

Finalizada la simulación del transporte, luego de las 24 horas, se registró un $100 \%$ de sobrevivencia en los tratamientos $15 \mathrm{C}-0,15 \mathrm{C}$ $15,19 \mathrm{C}-0$ y $19 \mathrm{C}-15$. Los tratamientos $15 \mathrm{C}-$ $20,15 \mathrm{C}-25$ y $19 \mathrm{C}-20$ también presentaron altos porcentajes de sobrevivencia con el 95, 67 y $71 \%$, respectivamente; sin embargo, la sobrevivencia se vio afectada en el tratamiento $19 \mathrm{C}-25$, registrándose solo el $29 \%$ del total de individuos (Figura 2c). 

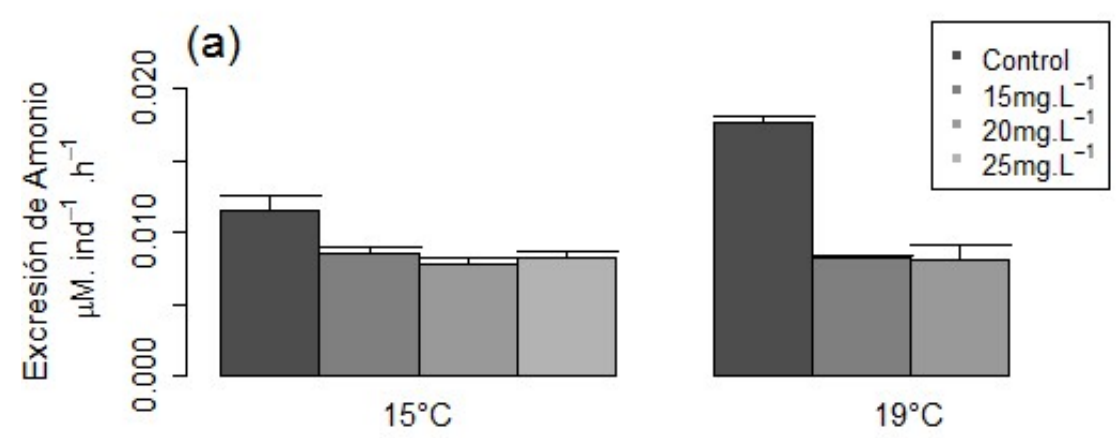

(b)
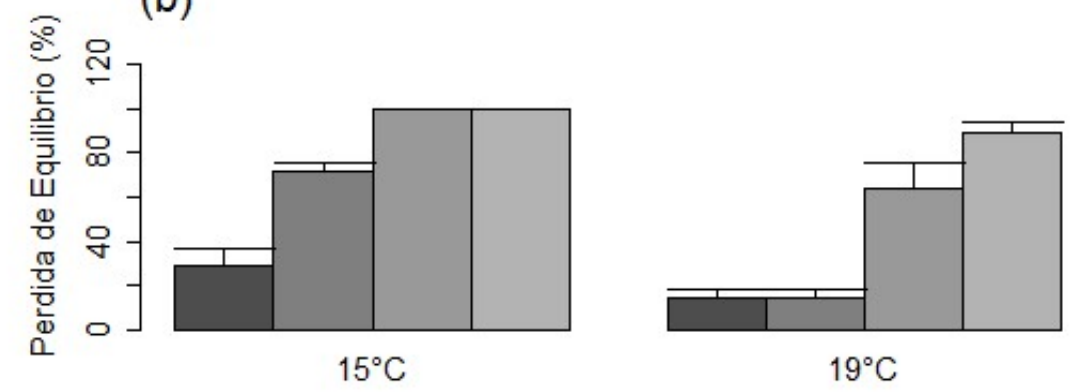

(c)
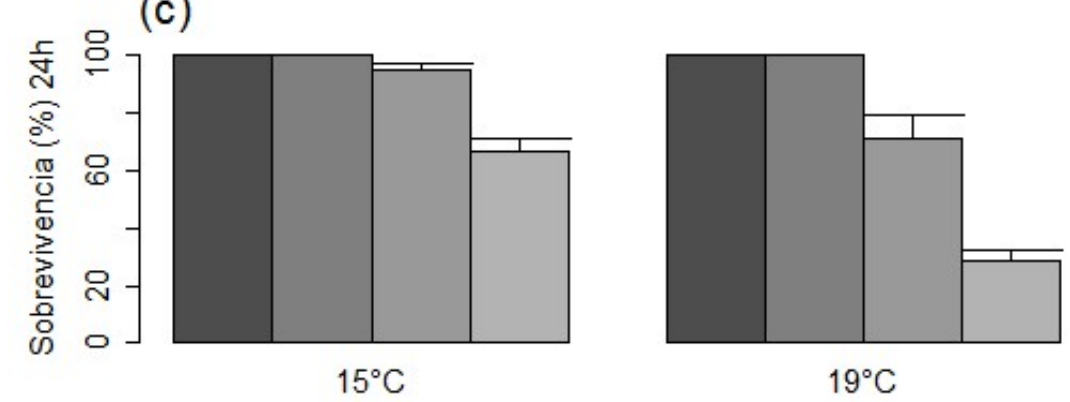

$19^{\circ} \mathrm{C}$

(d)
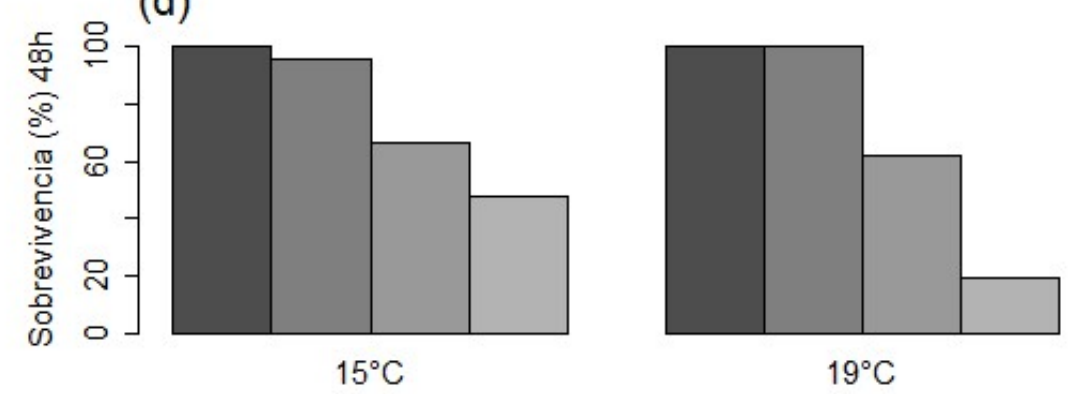

Figura 2. Resultados de la prueba de simulación de transporte para juveniles de Chita mostrando (a) índices de tasa de excreción de amonio individual; (b) Pérdida de Equilibrio; (c) Sobrevivencia 24 h; (d) Sobrevivencia $48 \mathrm{~h}$ 
Cuadro 4. Índices de la simulación de transporte con uso de tricaína (MS-222: 0, 15, 20 y $25 \mathrm{mg} / \mathrm{L}$ ) a 15 y $19^{\circ} \mathrm{C}$ para juveniles de chita

\begin{tabular}{cccccc}
\hline Tratamiento & $\begin{array}{c}\text { Excreción de } \\
\text { amonio }\end{array}$ & $\mathrm{PE}^{1}$ & \multicolumn{2}{c}{ Sobrevivencia } & Resultado \\
\cline { 4 - 6 } $15 \mathrm{C}-0$ & 0 & 1 & $24 \mathrm{~h}$ & $48 \mathrm{~h}$ & \\
$15 \mathrm{C}-15$ & 1 & 0 & 1 & 1 & 3 \\
$15 \mathrm{C}-20$ & 1 & 0 & 0 & 0 & 2 \\
$15 \mathrm{C}-25$ & 1 & 0 & 0 & 0 & 1 \\
$19 \mathrm{C}-0$ & 0 & 1 & 1 & 1 & 1 \\
$19 \mathrm{C}-15$ & 1 & 1 & 1 & 1 & 3 \\
$19 \mathrm{C}-20$ & 1 & 0 & 0 & 0 & 4 \\
$19 \mathrm{C}-25$ & 0 & 0 & 0 & 0 & 1 \\
\hline
\end{tabular}

${ }^{1}$ Pérdida de equilibrio

Al término de las 48 horas, incluyendo el periodo de recuperación, la sobrevivencia en los tratamientos 15C-15, 19C-0 y 19C-15 se mantuvo en $100 \%$. En los tratamientos $15 \mathrm{C}-15,15 \mathrm{C}-20,15 \mathrm{C}-25$ y $19 \mathrm{C}-201 \mathrm{a}$ sobrevivencia disminuyó a 95, 67, 48 y 62\%, respectivamente. En el tratamiento 19C-25 se registró el porcentaje de sobrevivencia más bajo con 19\% del total de individuos (Figura 2d).

\section{Simulación del Transporte}

Los resultados de la evaluación de la simulación del transporte (Cuadro 4) muestran que el tratamiento 19C-15 alcanza un puntaje final de 4 , haciéndolo el tratamiento más adecuado para el transporte de juveniles de chita en comparación con el resto de condiciones evaluadas. Los tratamientos $15 \mathrm{C}-0$ y 19C-0 obtuvieron un puntaje adecuado de 3 , de modo que también podrían considerarse como una alternativa viable. Los demás tratamientos (15C-15, 15C-20, 15C-25, 19C20 y $19 \mathrm{C}-25)$ presentan valores menores que 3 , considerándose como no recomendables.

\section{Discusión}

Las concentraciones de tricaína (MS222) utilizadas durante la prueba preliminar fueron inicialmente propuestas usando como referencia las utilizadas por Schoetteger y Julian (1967) en transporte de salmón. Sin embargo, estas concentraciones podrían variar según la especie y tamaño del pez, así como la temperatura y la dureza del agua (Cho y Heath, 2000; Hinostroza y Serrano, 2013). En la prueba preliminar se determinó que concentraciones de tricaína mayores de $26 \mathrm{mg} . \mathrm{L}^{-1}$ no serían recomendables; pues según Summerfelt y Smith (1990), una sedación profunda con pérdida de equilibrio no es deseable en el transporte de peces debido al incremento de riesgo de lesiones físicas y de asfixia (acumulación de peces en el fondo y esquinas del recipiente de transporte). Con este criterio se fijó la concentración máxima de tricaína en $25 \mathrm{mg} . \mathrm{L}^{-1}$ para las prueba de transporte simulado en bolsas con inyección de oxígeno. 
Las temperaturas fueron establecidas tomando el rango de tolerancia de la chita, el cual está entre 11 y $32^{\circ} \mathrm{C}$ (IMARPE, 2014). En estos casos, el tratamiento a $19^{\circ} \mathrm{C}$ buscó simular una temperatura ambiental (transporte simple y menos costoso) y el tratamiento a $15^{\circ} \mathrm{C}$ simuló una temperatura con tendencia al límite bajo de tolerancia (disminución del metabolismo).

Las mediciones de $\mathrm{pH}$ al interior de las bolsas de transporte muestran una disminución importante al término de las 24 horas de simulación. Una disminución del $\mathrm{pH}$ por efecto de la disolución de la tricaína en el agua ha sido reportado por Velasco et al. (2008) y Guo et al. (1995). Sin embargo, Oikawa et al. (1994) afirman que cuando la MS-222 es disuelta en agua de mar a concentraciones entre 50 y 100 ppm, el pH de la solución resultante estaría en un rango neutro (7.72 7.15), debido a la presencia de minerales carbonatados, generando un efecto de amortiguación sobre el $\mathrm{pH}$ del agua (Barceló et al., 2002). Además, Carter et al. (2011) mencionan que el agua de mar o el agua dulce con alta alcalinidad contienen suficientes amortiguadores para mantener el $\mathrm{pH}$ en un rango aceptable. Por otro lado, Woynarovich y Horváth (1981) mencionan que la disminución del $\mathrm{pH}$ es debido al $\mathrm{CO}_{2}$ producido por la respiración de los peces. En ese sentido, resultados de consumo de oxígeno muestran que la tasa de respiración de $A$. scapularis es relativamente elevada (IMARPE, 2014), siendo entonces la producción de $\mathrm{CO}_{2}$ la causa más probable de disminución de $\mathrm{pH}$ en las bolsas de transporte. Por otro lado, es posible la adición de buffers para mantener el $\mathrm{pH}$ del agua de transporte en valores seguros (Hettiarachchi y Senadheera, 1999). En este caso, se deberá considerar la biomasa de peces a transportar, la temperatura y las condiciones de transporte en general.

El uso de anestésico favorece la disminución de la excreción de los peces durante el transporte (Bower y Turner, 1982). La tendencia observada en el presente estudio corrobora estas observaciones, donde los tra- tamientos con MS-222 mostraron menores tasas de excreción individual en ambas temperaturas, coincidiendo con Guo et al. (1995). Sin embargo, en los tratamientos sin tricaína, se observó que las concentraciones de amonio fueron mayores a $19^{\circ} \mathrm{C}$, debido a que la temperatura, como en otros ectotermos, aumenta el metabolismo de los peces, haciendo que excreten mayor cantidad de desechos metabólicos (Kubitza, 2009). En ese sentido, cabe resaltar que, si bien, a $15^{\circ} \mathrm{C}$ sin anestésico, la excreción es relativamente baja, se obtuvieron excreciones similares con el uso de tricaína a $19{ }^{\circ} \mathrm{C}$. Esto confirma la capacidad del anestésico utilizado para reducir la actividad metabólica de $A$. scapularis durante la simulación de transporte.

Por otro lado, Hettiarachchi y Senadheera (1999) mencionan que el problema de la acumulación de amonio en el transporte de peces se debe principalmente a la alimentación. Peces en inanición por un periodo de 2 a 3 días, dependiendo su peso corporal, disminuirán considerablemente su tasa de excreción. En este caso, los peces no fueron alimentados por un periodo 24 horas; sin embargo, al finalizar la simulación se observaron heces en el fondo de las bolsas. Con base a estos resultados, sería recomendable realizar los transportes con un periodo de inanición superior a 24 horas.

Un mayor porcentaje de pérdida de equilibrio fue observado en juveniles de chita transportados a $15^{\circ} \mathrm{C}$ en comparación con $19^{\circ} \mathrm{C}$. Esto coincide con otros resultados (Schoetteger y Julian, 1967; Smith y Bell, 1967) en salmónidos, donde un mayor efecto sedante fue registrado a temperaturas más bajas. Hikasa et al. (1986) indican que la temperatura influye en el estado de la anestesia y en el tiempo de recuperación, mientras que Schoetteger y Julian (1967) indican que la anestesia se combina con el efecto del letargo del animal cuando es colocado a temperaturas bajas. Un transporte a bajas temperaturas requerirá, por lo tanto, de concentraciones de anestésico más bajas para evitar el incremento de la frecuencia de pérdida de equilibrio. 
Existen muchas alternativas de anestésicos en el mercado, como la benzocaina, lidocaina, quinaldina (Coyle et al., 2004) y eugenol, entre otros; sin embargo, el MS-222 (metanosulfonato de triciana) es el único aprobado por la Food and Drug Administration (FDA) para uso en animales de consumo humano. Además, Hikasa et al. (1986) indican que la predilección de la MS222 se debe a la rápida inducción y recuperación que muestran los peces.

\section{Conclusiones}

- El uso de una dosis baja de tricaína (15 mg.L $\left.{ }^{-1}\right)$ mejora las condiciones de transporte de juveniles de chita a temperatura ambiental $\left(19^{\circ} \mathrm{C}\right)$.

- La disminución de la temperatura incrementa el efecto sedante de la tricaína en juveniles de chita.

- El uso de tricaína en el transporte de juveniles de chita $(6-9 \mathrm{~cm})$ no deberá superar los $20 \mathrm{mg} \cdot \mathrm{L}^{-1}$.

\section{Agradecimientos}

Los autores agradecen al PpR «Ordenamiento y Desarrollo de la Acuicultura» (DGIA-IMARPE) por el financiamiento del presente proyecto de investigación. Asimismo, se agradece el valioso apoyo de los colegas del Laboratorio de Cultivos Marinos (IMARPE) que proporcionaron los ejemplares para la ejecución del experimento.

\section{Literatura Citada}

1. Allen GR, Robertson DR. 1994. Fishes of the tropical Eastern Pacific. Honolulu, USA: University of Hawaii Press. 332 p.

2. Barceló I, Allende I, Solis H, Bussy A, González C. 2002. Determinación del estado de equilibrio de un sistema hídrico. Rev Soc Quím Méx 46: 93-104.

3. Bower C, Turner D. 1982. Ammonia removal by Clinoptilolite in the transport of ornamental freshwater fishes. Prog Fish Cult 44: 19-23. doi: 10.1577/15488659(1982)44[19:ARBCIT]2.0.CO;2

4. Carter K, Woodley C, Brown R. 2011. A review of tricaine methanesulfonate for anesthesia of fish. Rev Fish Biol Fisheries 21:51-59. doi: 10.1007/s11160010-9188-0

5. Chero J, Cruces C, Iannacone J, Saez G, Alvariño L. 2014. Helmintos parásitos de Anisotremus scapualris (Tschudi, 1846) (Perciformes: Haemulidae) «Chita» adquiridos en el terminal pesquero de Villa María del Triunfo, Lima, Perú. Neotrop Helminthol 8: 411-428.

6. Chirichigno N, Vélez J. 1998. Clave para identificar los peces marinos del Perú. $2^{\mathrm{a}}$ ed. Perú: Instituto del Mar del Perú. 496 p.

7. Cho GK, Heath DD. 2000. Comparison of tricaine methanesulphonate (MS22) and clove oil anaesthesia effects on the physiology of juvenile chinook salmon Oncorhinchus tshawytscha (Walbaum). Aquac Res 31: 537-546. doi: 10.1046/j.1365-2109.2000.00478.x

8. Coyle S, Durborow R, Tidwell J. 2004. Anesthetics in aquaculture. SRAC Publication No. 3900. Texas, USA: Southern Regional Aquaculture Center. $6 \mathrm{p}$.

9. [FAO] Organización de las Naciones Unidas para la Alimentación y la Agricultura. 2010. Perfiles sobre la pesca y la acuicultura por países: la Republica del Perú. [Internet]. Disponible en: http://www.fao.org/fishery/facp/ PER/es

10. Guo F-C, Teo L-H, Chen T-W. 1995. Effects of anaesthetics on the water parameters in a simulated transport experiment of platyfish, Xiphophorus maculatus (Günther). Aquaculture Res 26: 265-271. doi: $10.1111 / \mathrm{j} .1365-$ 2109.1995.tb00911.x

11. Hettiarachchi M, Senadheera SPSD. 1999. Efficacy of quinaldine sulphato as an anaesthetic for the ornamental carp (Cyprinus carpio) in simulated packaging for long distance transport. Sri Lanka J Aquat Sci 4: 13-22. 
12. Hikasa Y, Takase K, Ogasawara T, Ogasawara S. 1986. Anesthesia and recovery with tricaine methanesulfonate, eugenol and thiopental sodium in the carp, Cyprinus carpio. Jpn J Vet Sci 48: 341-351. doi: 10.1292/jvms 1939. 48.341

13. Hinostroza E, Serrano E. 2013. Efecto anestésico del metasulfonato de tricaína en paiches (Arapaima gigas) juveniles. Rev Inv Vet Perú 24: 451-458. doi: 10.15381/rivep.v24i4.2736

14. [IMARPE] Instituto del Mar del Perú. 2014. Anuario Científico Tecnológico: evaluaciones ecofisiologicas en juveniles de chita y cabrilla. Vol 14. $146 \mathrm{p}$.

15. Iannacone J, Alvariño L. 2009. Aspectos cuantitativos de la parasitofauna de Anisotremus scapularis (Tschudi) (Osteichthyes, Haemulidae) capturados por pesquería artesanal en Chorrillos, Lima, Perú. Rev Ibero-Latinoam Parasitol 68: 56-64.

16. Iannacone J, Alvariño L. 2012. Microecology of the monogenean Mexicana sp on the gills of Anisotremus scapularis (Tschudi, 1846) (Osteichthyes, Haemulidae) of the marine coast of Lima, Peru. Neotrop Helminthol 6: 277-285.

17. Kubitza F. 2009. Manejo en la producción de peces: buenas prácticas en el transporte de peces vivos. Panorama da Aquicultura. [Internet]. Disponible en: http://www.agroindustria.gob.ar/site/pesca/acuicultura/06_Publicaciones/_archivos $/ 091230 \_$Manejo\%20en\%201a\% 20producci\%C3\%B3n\%20de\%20peces.pdf

18. Medina M, Araya M, Vega C. 2004. Alimentación y relaciones tróficas de peces costeros de la zona norte de Chile. Invest Mar 32: 33-47. doi: 10.4067/ S0717-71782004000100004

19. [PRODUCE] Ministerio de la Producción. 2015. Anuario estadístico pesquero y acuícola 2013. Perú: Produ- ce. 113 p. [Internet]. Disponible en: http:/ /www.produce.gob.pe/images/stories/ Repositorio/estadistica/anuario/anuarioestadistico-pesca-2013.pdf

20. Oikawa S, Takeda T, Itazawa Y. 1994. Scale effects of MS-222 on a marine teleost, porgy Pagrus major. Aquaculture 121:369-379. doi: 10.1016/ 0044-8486(94)90271-2

21. Ruelas N, Córdova B. 1995. Monogeneos de Scomber japonicus peruanus del litoral sur peruano. Rev Peru Parasitol 11(1): 61-64.

22. Sato T, Kitayama S, Mitsuhata C, Ikeda T, Morita K, Dohi T. 2000. Selective inhibition of monoamine neurotransmitter transport by synthetic local anesthetics. Naunyn-Schmiedeberg's Arch Pharmacol 361: 214-220. doi:10.1007/s002109900184

23. Schoettger R, Julian A. 1967. Efficacy of MS-222 as an anesthetic on four salmonids. USA: US Fish and Wildlife Service. $15 \mathrm{p}$.

24. Smith L, Bell G. 1967. Anesthetic and surgical techniques for pacific salmon. J Fish Res Board Canada 24: 1579-1588. doi: 10.1139/f67-130

25. Summerfelt R, Smith L. 1990. Anaesthesia, surgery and related techniques. In: Schreck C, Moyle P (eds). Methods for fish biology. USA: American Fisheries Society. p 213-272.

26. Woynarovich E, Horváth L. 1981. Propagación artificial de peces de aguas templadas: manual para extensionistas. FAO, Documentos Técnicos de Pesca 201. Rome: FAO. 187 p.

27. Velasco Y, Palacios C, Cruz P. 2008. Eficiencia anestésica de 2-fenoxietanol, benzocaína, quinaldina y metasulfonato de tricaína en alevinos y juveniles de cachama blanca (Piaractus brachypomus). Rev MVZ Córdoba 13: 14351445. 